This article is licensed under the Creative Commons Attribution-NonCommercial 4.0 International License (CC BY-NC) (http://www.karger.com/Services/OpenAccessLicense). Usage and distribution for commercial purposes requires written permission.

\title{
Difficulty Achieving a Preoperative Diagnosis of IgG4-Related Sclerosing Cholangitis
}

\author{
Kazuhiro Suzumura ${ }^{a}$ Etsuro Hatano ${ }^{a}$ Masaharu Tada ${ }^{a}$ \\ Hideaki Sueoka ${ }^{a}$ Hiroshi Nishida $^{a}$ Kenjiro lida ${ }^{a}$ Seikan Hai ${ }^{a}$ \\ Hayato Miyamoto $^{b}$ Tatsuya Andoh ${ }^{c}$ Takahiro Ueki ${ }^{d}$ Kentaro Nonaka \\ Keiji Nakasho ${ }^{f}$ Jiro Fujimoto ${ }^{a}$
}

aDepartment of Surgery, Hyogo College of Medicine, Nishinomiya, Japan; bepartment of Internal Medicine, Takarazuka City Hospital, Takarazuka, Japan; 'Department of Surgery, Takarazuka Daiichi Hospital, Takarazuka, Japan; dDepartment of Surgery, Ueki Hospital, Osaka, Japan; eDepartment of Surgery, Higashinari Hospital, Osaka, Japan; fDepartment of Pathology, Hyogo College of Medicine, Nishinomiya, Japan

\section{Keywords}

IgG4-related sclerosing cholangitis · Bile duct cancer · Autoimmune pancreatitis

\begin{abstract}
A 75-year-old male was admitted to our hospital because of bile duct stenosis. He had no medical history of autoimmune disease. The level of tumor markers, serum IgG, and IgG4 were within normal ranges. Computed tomography showed perihilar and distal bile duct stenosis and wall thickening without swelling or abnormal enhancement of the pancreas. Endoscopic retrograde cholangiopancreatography showed perihilar and distal bile duct stenosis. A biopsy and cytology from the distal bile duct stenosis suggested adenocarcinoma, and cytology from the perihilar bile duct also suggested adenocarcinoma. A preoperative diagnosis of perihilar and distal bile duct cancer was made, and the patient underwent left hepatectomy and
\end{abstract}




\section{Case Reports in Gastroenterology}

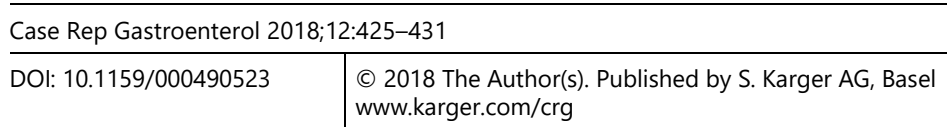
www.karger.com/crg

Suzumura et al.: IgG4-Related Sclerosing Cholangitis

pancreaticoduodenectomy. Resected specimens showed wall thickening in the perihilar and distal bile duct; however, tumors were unclear. A histopathological examination revealed lymphoplasmacytic infiltration, storiform fibrosis, and obliterative phlebitis in the perihilar and distal bile ducts. Immunohistochemistry revealed diffuse infiltration of IgG4-positive plasma cells in the perihilar and distal bile ducts. Lymphoplasmacytic infiltration, inflammatory change, storiform fibrosis, and obliterative phlebitis were shown in the pancreas. A final diagnosis of IgG4-related sclerosing cholangitis (IgG4-SC) with autoimmune pancreatitis was made. We herein report a case in which a preoperative diagnosis of IgG4-SC was difficult due to normal serum lgG4 levels and no obvious pancreatic lesion.

(C) 2018 The Author(s)

Published by S. Karger AG, Basel

\section{Background}

IgG4-related sclerosing cholangitis (IgG4-SC) occurs mainly in elderly patients in association with IgG4-positive plasma cell infiltration of tissue, leading to fibrosis of various organs, such as the pancreas, salivary glands, bile duct, and retroperitoneum [1]. IgG4-SC is often accompanied by obstructive jaundice due to bile duct stricture and is usually associated with autoimmune pancreatitis (AIP) and elevated serum IgG4 levels [2]. It is important to diagnose IgG4-SC before surgery to avoid any unnecessary surgical resection because steroid therapy is effective in these cases [3]. However, IgG4-SC is difficult to distinguish from bile duct cancer if the serum IgG4 levels are normal and pancreatic imaging findings are absent.

We herein report a case in which a preoperative diagnosis of IgG4-SC was difficult due to normal serum IgG4 levels and no obvious pancreatic lesion.

\section{Case Presentation}

A 75-year-old male visited another hospital because of jaundice. Computed tomography (CT) revealed intrahepatic bile duct dilatation. He underwent endoscopic retrograde cholangiopancreatography (ERCP) and received an endoscopic retrograde biliary drainage tube in the common bile duct (CBD) for the distal bile duct stenosis.

He was then referred to our hospital for a further inspection and treatment. He had a history of treatment for bladder cancer. He had no medical history of autoimmune disease. On a laboratory examination, the serum levels of total bilirubin, alkaline phosphatase, $\gamma$-glutamyl transpeptidase, and amylase were within normal ranges. The levels of the tumor markers carcinoembryonic antigen, carbohydrate antigen 19-9, S-pancreas- 1 antigen, and DUPAN-2 were also within normal ranges. An immunological examination revealed that the levels of serum $\mathrm{IgG}=966 \mathrm{mg} / \mathrm{dL}$ (normal range 870-1700) and IgG4 = 78.6 mg/dL (normal range 4.5-117) were within normal ranges as well (Table 1). Perinuclear antineutrophilic cytoplasmic antibody, antimitochondrial antibody, and antinuclear antibody were negative.

CT showed perihilar and distal bile duct stenosis and wall thickening without swelling or abnormal enhancement of the pancreas (Fig. 1a). Magnetic resonance cholangiopancreatography showed perihilar and distal bile duct stenosis, but the main pancreatic duct was normal (Fig. 1b). Fluorodeoxyglucose positron emission tomography showed a slight uptake by the 
distal bile duct (maximum standard uptake value: 3.7). No other sites, including the perihilar bile duct, showed any FDG uptake. Intraductal ultrasonography showed circular-symmetric wall thickening at the perihilar and distal bile ducts (Fig. 1c, d). ERCP showed perihilar and distal bile duct stenosis (Fig. 1e). A biopsy and cytology analysis from the distal bile duct stenosis suggested adenocarcinoma, and cytology from the perihilar bile duct also suggested adenocarcinoma.

Based on these findings, a preoperative diagnosis of perihilar and distal bile duct cancer was made, and the patient underwent left hepatectomy and pancreaticoduodenectomy. The resected specimens showed wall thickening in the perihilar and distal bile ducts; however, tumors were unclear (Fig. 2a, b).

A histopathological examination revealed lymphoplasmacytic infiltration, storiform fibrosis, and obliterative phlebitis in the perihilar and distal bile duct (Fig. 3a, b). Immunohistochemistry revealed the diffuse infiltration of IgG4-positive plasma cells in the perihilar and distal bile ducts (Fig. 3c, d). Lymphoplasmacytic infiltration, inflammatory change, storiform fibrosis, and obliterative phlebitis were shown in the pancreas (Fig. 3e). Immunohistochemistry revealed diffuse infiltration of IgG4-positive plasma cells in the pancreas (Fig. 3f). A final diagnosis of IgG4-SC with AIP was made based on the histological and immunohistochemical findings.

The patient was discharged from the hospital on the 39th day after surgery. He has remained well for 3 months since undergoing surgery.

\section{Discussion}

IgG4-related disease is a fibroinflammatory, multiorgan condition of unknown etiology with commonly shared features that include elevated serum IgG4 concentrations, tumefactive lesions, storiform fibrosis, and dense lymphocyte and IgG4-positive plasmacyte infiltrates [4]. The disease usually responds well to steroid therapy [4].

IgG4-SC is often accompanied by obstructive jaundice due to bile duct stricture and is usually associated with diffuse pancreatic enlargement or pancreatic mass with autoimmune pancreatitis. In patients with IgG4-SC, the serum IgG4 levels are often elevated. In 2012, clinical diagnostic criteria for IgG4-SC were proposed in Japan [5] as follows: (1) characteristic biliary imaging findings, (2) elevated serum IgG4 concentration, (3) coexisting IgG4-related disease except for that of the biliary tract, and (4) characteristic histopathological features. The present case showed stenoses of the bile duct but no elevated serum IgG4 concentrations or abnormal imaging findings of the pancreas. Similarly, some previous IgG4-SC cases were also reported to show no elevated serum IgG4 concentrations $[6,7]$ or no abnormal imaging findings of the pancreas $[8,9]$; it is therefore necessary to diagnose IgG4-SC carefully. Nakazawa et al. [10] classified IgG4-SC into 4 types when making a differential diagnosis based on the cholangiographic features: stricture located in the lower part of the CBD is type 1, stricture that is diffusely distributed in the intra- and extrahepatic bile ducts is type 2 , stricture distributed in both the hilar hepatic region and the lower part of the CBD is type 3 , and stricture of the bile duct in the hilar hepatic region is type 4. Type 3 and 4 IgG4-SC may mimic bile duct cancer and can therefore be difficult to differentiate. The patient described herein was a case 
suspected of being type 3 IgG4-SC; however, we diagnosed him with bile duct cancer because the brushing cytology of the bile duct revealed findings suggestive of adenocarcinoma.

Regarding bile duct brushing cytology for the diagnosis of carcinoma, the reported sensitivity of the technique for carcinoma has ranged from 35 to $75 \%$, and the specificity has ranged from 89 to $100 \%$ [11-13]. The diagnosis ability of bile duct brushing cytology is limited. Therefore, if suspected malignant cells are obtained from the bile duct stenosis site, the imaging findings should be rechecked, and the cytology and biopsy should be repeated to confirm the diagnosis.

Regarding the treatment of IgG4-SC, the disease usually responds well to steroid therapy. In the clinical diagnostic criteria for IgG4-SC, the effectiveness of steroid therapy is an optional additional diagnostic criterion to confirm the accurate diagnosis of IgG4-SC [5]. In the present case, to avoid delaying treatment of bile duct cancer, we did not administer steroid therapy. Moon et al. [14] conducted a 2-week steroid trial and subsequent assessment of its response, with findings suggesting that this therapy may be helpful in confirming the diagnosis of atypical imaging for AIP. Therefore, short-term steroid therapy should be considered in patients suspected of having atypical IgG4-SC.

In conclusion, IgG4-SC is difficult to distinguish from bile duct cancer if the serum IgG4 levels are normal and pancreatic imaging findings are absent. If IgG4-SC is suspected, we therefore should diagnose it carefully by a repeated biopsy or short-term steroid therapy to avoid unnecessary major surgery.

\section{Statement of Ethics}

Approval from the ethics committee was not required for this case report. Written informed consent was obtained from the patient for the publication of this case report and any accompanying images.

\section{Disclosure Statement}

None of the authors has any financial conflicts of interest to disclose.

\section{References}

1 Hamano H, Kawa S, Horiuchi A, Unno H, Furuya N, Akamatsu T, et al. High serum IgG4 concentrations in patients with sclerosing pancreatitis. N Engl J Med. 2001 Mar;344(10):732-8.

2 Nishino T, Toki F, Oyama H, Oi I, Kobayashi M, Takasaki K, et al. Biliary tract involvement in autoimmune pancreatitis. Pancreas. 2005 Jan;30(1):76-82.

3 Shimizu S, Naitoh I, Nakazawa T, Hayashi K, Miyabe K, Kondo H, et al. IgG4-related sclerosing cholangitis with no biliary stricture but severe thickening of the bile duct wall. Intern Med. 2016;55(12):1575-9.

4 Stone JH, Zen Y, Deshpande V. IgG4-related disease. N Engl J Med. 2012 Feb;366(6):539-51.

5 Ohara H, Okazaki K, Tsubouchi H, Inui K, Kawa S, Kamisawa T, et al.; Research Committee of IgG4-related Diseases; Research Committee of Intractable Diseases of Liver and Biliary Tract; Ministry of Health, Labor and Welfare, Japan; Japan Biliary Association. Clinical diagnostic criteria of IgG4-related sclerosing cholangitis 2012. J Hepatobiliary Pancreat Sci. 2012 Sep;19(5):536-42. 


\section{Case Reports in Gastroenterology} \begin{tabular}{l|l}
\hline Case Rep Gastroenterol 2018;12:425-431 \\
\hline DOI: 10.1159/000490523 & $\begin{array}{l}\text { ○ 2018 The Author(s). Published by S. Karger AG, Basel } \\
\text { www.karger.com/crg }\end{array}$
\end{tabular}

Suzumura et al.: IgG4-Related Sclerosing Cholangitis

6 Mizutani S, Suzuki H, Yoshida H, Arima Y, Kitayama Y, Uchida E. Case of IgG4-related sclerosing cholangitis with a normal serum IgG4 level: report of a case. J Nippon Med Sch. 2012;79(5):367-72.

7 Nakazawa T, Ikeda Y, Kawaguchi Y, Kitagawa H, Takada H, Takeda Y, et al. Isolated intrapancreatic IgG4related sclerosing cholangitis. World J Gastroenterol. 2015 Jan;21(4):1334-43.

8 Matsubayashi H, Uesaka K, Sugiura T, Ohgi K, Sasaki K, Ono H. IgG4-related sclerosing cholangitis without obvious pancreatic lesion: difficulty in differential diagnosis. J Dig Dis. 2014 Jul;15(7):394-403.

9 Graham RP, Smyrk TC, Chari ST, Takahashi N, Zhang L. Isolated IgG4-related sclerosing cholangitis: a report of 9 cases. Hum Pathol. 2014 Aug;45(8):1722-9.

10 Nakazawa T, Ohara H, Sano H, Ando T, Joh T. Schematic classification of sclerosing cholangitis with autoimmune pancreatitis by cholangiography. Pancreas. 2006 Mar;32(2):229.

11 Rabinovitz M, Zajko AB, Hassanein T, Shetty B, Bron KM, Schade RR, et al. Diagnostic value of brush cytology in the diagnosis of bile duct carcinoma: a study in 65 patients with bile duct strictures. Hepatology. 1990 Oct;12(4 Pt 1):747-52.

12 Kurzawinski T, Deery A, Dooley J, Dick R, Hobbs K, Davidson B. A prospective controlled study comparing brush and bile exfoliative cytology for diagnosing bile duct strictures. Gut. 1992 Dec;33(12):1675-7.

13 Layfield LJ, Cramer H. Primary sclerosing cholangitis as a cause of false positive bile duct brushing cytology: report of two cases. Diagn Cytopathol. 2005 Feb;32(2):119-24.

14 Moon SH, Kim MH, Park DH, Hwang CY, Park SJ, Lee SS, et al. Is a 2-week steroid trial after initial negative investigation for malignancy useful in differentiating autoimmune pancreatitis from pancreatic cancer? A prospective outcome study. Gut. 2008 Dec;57(12):1704-12.
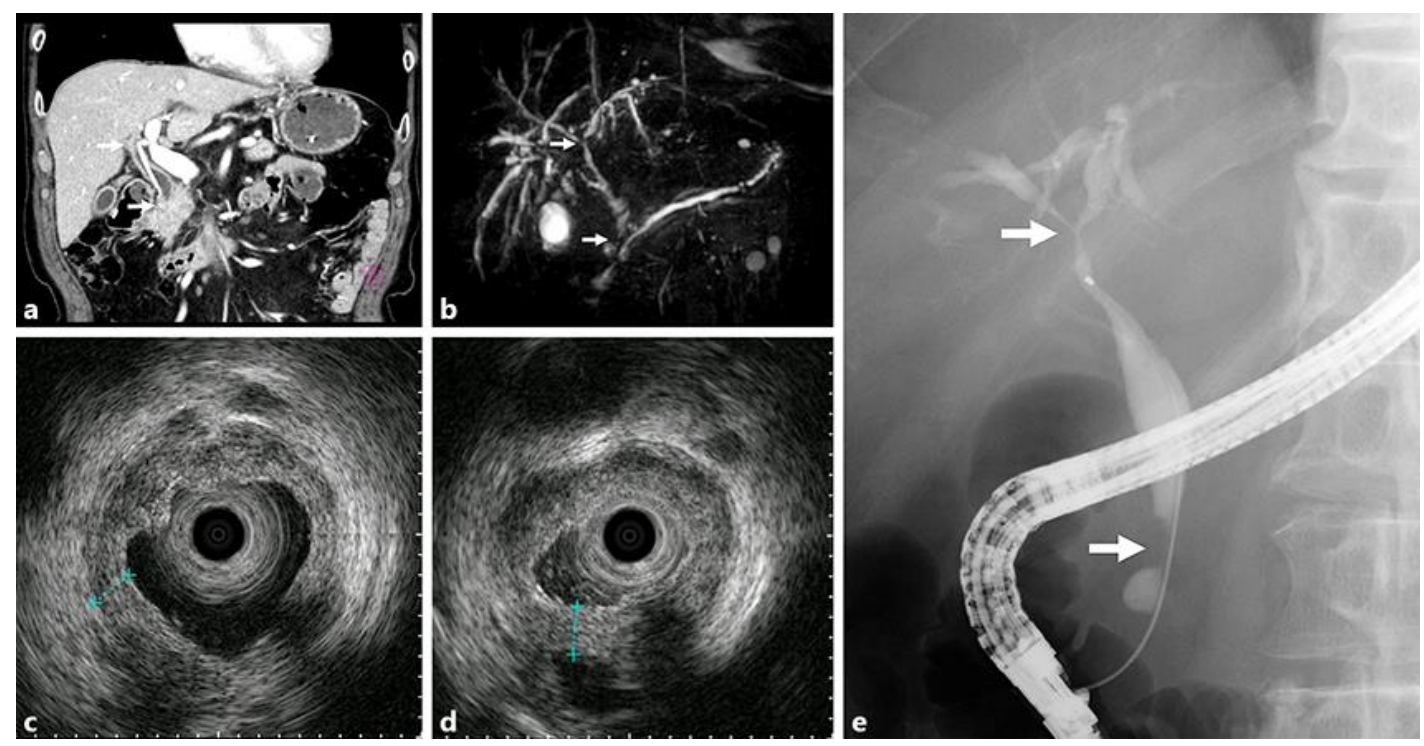

Fig. 1. CT images showed perihilar and distal bile duct stenosis and wall thickening (arrows) without swelling or abnormal enhancement of the pancreas (a). Magnetic resonance cholangiopancreatography showed perihilar and distal bile duct stenosis (arrows), but the main pancreatic duct was normal (b). Intraductal ultrasonography showed circular-symmetric wall thickening at the perihilar and distal bile ducts (c perihilar, d distal). ERCP showed perihilar and distal bile duct stenosis (arrows) (e). 


\section{Case Reports in Gastroenterology}

Case Rep Gastroenterol 2018;12:425-431
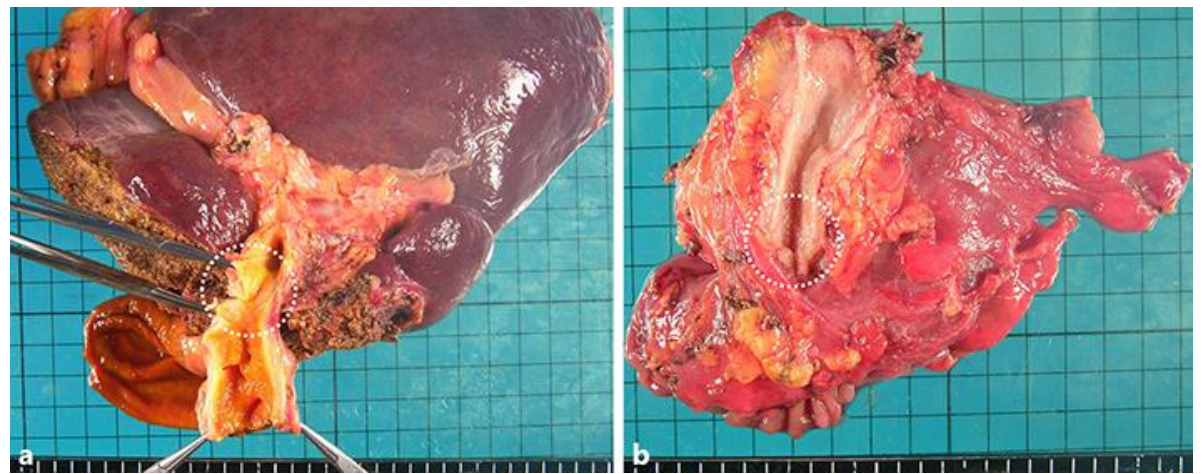

Fig. 2. Resected specimens showed wall thickening in the perihilar and distal bile duct; however, tumors were unclear (a perihilar, b distal).
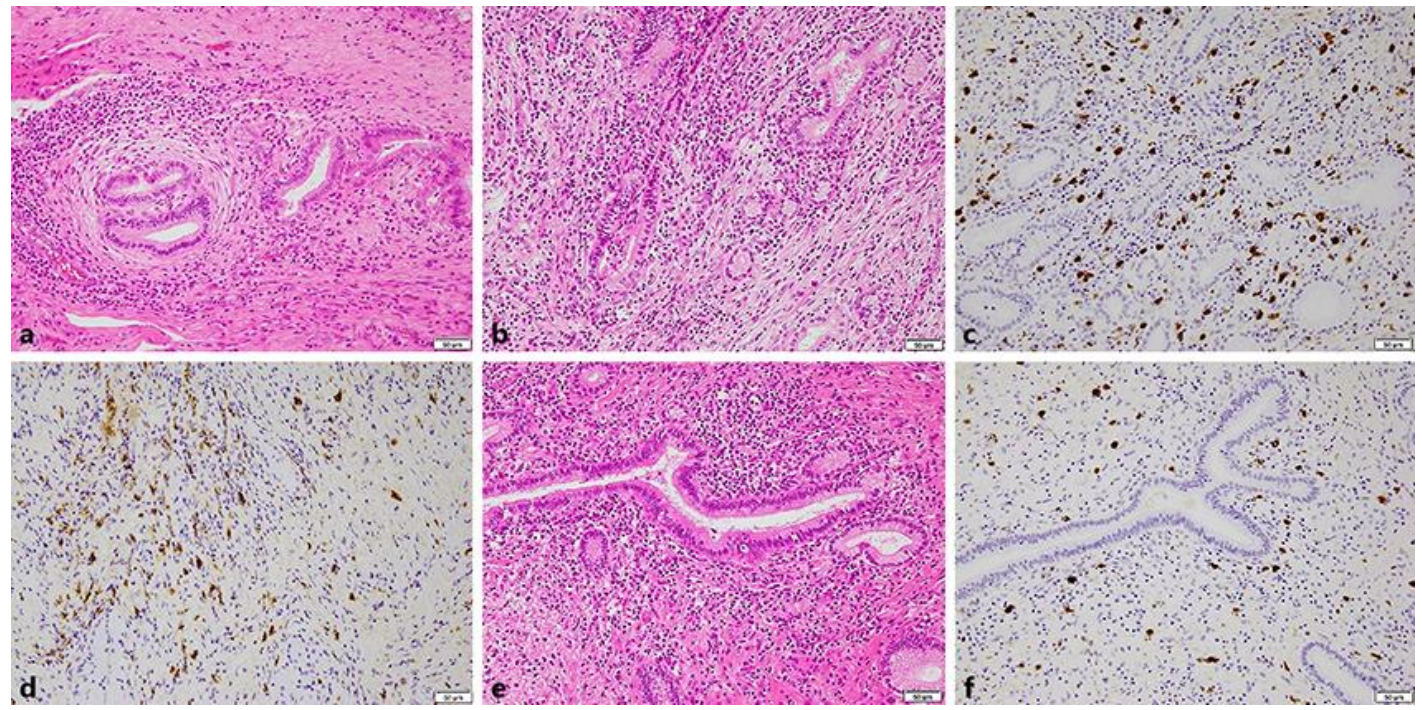

Fig. 3. A histopathological examination revealed lymphoplasmacytic infiltration, storiform fibrosis, and obliterative phlebitis in the perihilar and distal bile duct $(\mathbf{a}$ perihilar $\times 200$, $\mathbf{b}$ distal $\times 200$ ). Immunohistochemistry revealed diffuse infiltration of IgG4-positive plasma cells in the perihilar and distal bile ducts (c perihilar $\times 200$, d distal $\times 200$ ). Lymphoplasmacytic infiltration, inflammatory change, storiform fibrosis, and obliterative phlebitis were shown in the pancreas (e) $(\times 200)$. Immunohistochemistry revealed diffuse infiltration of IgG4-positive plasma cells in the pancreas $(f)(\times 200)$. 
Table 1. Laboratory data on admission

\begin{tabular}{llll}
\hline WBC & $4,790 / \mu \mathrm{L}$ & $\mathrm{Na}$ & $141 \mathrm{mmol} / \mathrm{L}$ \\
$\mathrm{RBC}$ & $426 \times 10^{4} / \mu \mathrm{L}$ & $\mathrm{K}$ & $4.4 \mathrm{mmol} / \mathrm{L}$ \\
$\mathrm{Hb}$ & $13.4 \mathrm{~g} / \mathrm{dL}$ & $\mathrm{Cl}$ & $109 \mathrm{mmol} / \mathrm{L}$ \\
$\mathrm{Ht}$ & $40.2 \%$ & $\mathrm{BUN}$ & $20 \mathrm{mg} / \mathrm{dL}$ \\
$\mathrm{Plt}$ & $17.1 \times 10^{4} / \mu \mathrm{L}$ & Cre & $0.78 \mathrm{mg} / \mathrm{dL}$ \\
& & Amy & $71 \mathrm{U} / \mathrm{L}$ \\
$\mathrm{TP}$ & $6.8 \mathrm{~g} / \mathrm{dL}$ & $\mathrm{CRP}$ & $0.12 \mathrm{mg} / \mathrm{dL}$ \\
$\mathrm{Alb}$ & $4.2 \mathrm{~g} / \mathrm{dL}$ & PT & $87.5 \%$ \\
$\mathrm{~T}-\mathrm{Bil}$ & $0.4 \mathrm{mg} / \mathrm{dL}$ & & \\
AST & $16 \mathrm{U} / \mathrm{L}$ & CEA & $2.9 \mathrm{ng} / \mathrm{mL}$ \\
$\mathrm{ALT}$ & $12 \mathrm{U} / \mathrm{L}$ & $\mathrm{CA} 19-9$ & $14.5 \mathrm{U} / \mathrm{mL}$ \\
$\mathrm{LDH}$ & $159 \mathrm{U} / \mathrm{L}$ & Span -1 & $9.9 \mathrm{U} / \mathrm{mL}$ \\
$\mathrm{ChE}$ & $101 \mathrm{U} / \mathrm{L}$ & DUPAN-2 & $\leq 25 \mathrm{U} / \mathrm{mL}$ \\
$\mathrm{ALP}$ & $311 \mathrm{U} / \mathrm{L}$ & & \\
$\gamma-\mathrm{GTP}$ & $27 \mathrm{U} / \mathrm{L}$ & IgG & $966 \mathrm{mg} / \mathrm{dL}$ \\
FBS & $105 \mathrm{mg} / \mathrm{dL}$ & IgG- 4 & $78.6 \mathrm{mg} / \mathrm{dL}$ \\
\hline
\end{tabular}

WBC, white blood cells; RBC, red blood cells; Hb, hemoglobin; Ht, hematocrit; Plt, platelets; TP, total protein; Alb, albumin; T-Bil, total bilirubin; AST, aspartate transaminase; ALT, alanine aminotransferase; LDH, lactate dehydrogenase; ChE, cholinesterase; ALP, alkaline phosphatase; $\gamma$-GTP, gamma-glutamyl transpeptidase; FBS, fasting blood sugar; Cre, creatinine; Amy, amylase; CRP, C-reactive protein; PT, prothrombin time; CEA, carcinoembryonic antigen; CA19-9, carbohydrate antigen 19-9; SPan-1, Spancreas-1 antigen. 